\title{
Prevalência de Transtornos Mentais Comuns entre Estudantes de Medicina da Universidade Regional de Blumenau (SC)
}

\author{
Prevalence of Common Mental Disorders in \\ Medical Students from Universidade Regional \\ de Blumenau - SC
}

\author{
Eduardo Otávio Grether \\ Mateus Ciola Becker \\ Helena Medina Menezes ${ }^{1 \odot}$ \\ Carlos Roberto de Oliveira Nunes ${ }^{I}$
}

\section{PALAVRAS-CHAVE \\ - Transtornos Mentais. \\ - Prevalência. \\ - Fatores de Risco.}




\section{KEY-WORDS}

- Mental Disorders.

- Prevalence.

- Risk Factors.

Recebido em 10/5/19

Aceito em 6/7/19

\begin{abstract}
Common mental disorders (CMDs) are increasingly frequent in the world's population and are less severe than other groups of mental disorders. They are associated with loss of quality of life, relationship problems and psychological suffering. The studies that investigate the prevalence of CMDs are aimed at the occupational, academic and health levels. Brazilian studies in the area of Mental Health carried out with medical students indicate that $30 \%$ and $44 \%$ of this group has some type of common mental disorder or symptom. The aim of the present study is to detect CMDs among medical students from Universidade Regional de Blumenau/SC. This is a cross-sectional study carried out in August 2017 with 340 students from the 1st to the 12th semester of School. The Self-Reporting Questionnaire (SRQ-20) was used for CMD screening. The prevalence of CMD was 50.9\%. Classic CMDs were associated $(p<0.05)$ with the mean daily workload of more than 12 hours, less than one hour of leisure per day, having a previous medical history of psychiatric illness, using antidepressants, never having attended or been discharged from psychotherapy and the use of alcohol and other substances. A higher rate of suspected CMD cases was found in the present sample than in other studies with students from other Brazilian Medical Schools. The presence of CMD during the internship cycle was similar to that observed in the early cycles of medical school, despite the presence of protective factors, as demonstrated in the literature. The university must direct its attention to health promotion activities, together with health services and the family, creating a psychological support network. For this purpose, it has subsidies to establish prevention and care actions directed at the mental health of medical students.
\end{abstract}

Aceito em 6/7/19

\section{INTRODUÇÃO}

Os transtornos mentais comuns (TMC), ou transtornos mentais menores, são os mais frequentes e menos graves entre os transtornos mentais, sendo relacionados a grande sofrimento mental, dificuldades nos relacionamentos e perda de qualidade de vida ${ }^{1}$. Os TMC englobam depressão não psicótica, ansiedade e sintomas somatoformes, como cansaço, esquecimento, irritação e diminuição da concentração².

Estudos sugerem que os TMC atinjam de $9 \%$ a $12 \%$ da população mundial ${ }^{3}$. Não existem pesquisas que apresentem os índices de prevalência de problemas mentais no âmbito nacional, mas se estima que a prevalência de TMC no Brasil seja de $12 \%$ a $15 \%$ em todas as faixas etárias ${ }^{3}$. As faculdades de Medicina propiciam um ambiente estressor que pode prejudicar a qualidade de vida do estudante, seu desempenho acadêmico e bem-estar psicossocial, além de causar sofrimento mental e dificuldade de relacionamento ${ }^{4,5}$. A tensão presente na graduação médica inclui competição no processo de seleção do curso, necessidade de adquirir muitos conhecimentos, dificuldade na administração do tempo, individualismo, responsabilidades e expectativas sociais acerca do papel do médico ${ }^{1}$. Em quatro estudos transversais com uso do Self Reporting Questionnaire
(SRQ-20), as prevalências de TMC em estudantes de Medicina variaram de $33,6 \%$ a $44,7 \%{ }^{1,5-7}$.

A perda da saúde mental está relacionada com diminuição da produtividade do estudante, maior dificuldade de relacionamento e sofrimento mental ${ }^{5}$. Nesse sentido, a escola médica deve ter a capacidade de cuidar, respeitar, escutar e ajudar o estudante de Medicina a desenvolver mecanismos para lidar com a pressão a que estará submetido no cotidiano acadêmico e profissional, dando-lhe suporte psicológico e pedagógico ${ }^{9}$.

Este trabalho tem como objetivo identificar a prevalência de TMC entre os estudantes do curso de Medicina da Universidade Regional de Blumenau (Furb) e determinar associações com possíveis fatores de risco.

\section{MÉTODOS}

Foi realizado, entre agosto e outubro de 2017, estudo transversal com alunos do primeiro ao sexto ano do curso de Medicina da Universidade Regional de Blumenau (SC). Para participar da pesquisa, os alunos deviam estar regularmente matriculados no curso de Medicina e assinar o Termo de Consentimento Livre e Esclarecido (TCLE). A amostra do estudo foi composta por 340 alunos matriculados no curso de Medicina, entregan- 
do-se os questionários durante a aula no momento da pesquisa; os alunos da nona à décima segunda fase (internato) foram abordados no intervalo de suas atividades. Foram entregues 342 questionários, sendo que 340 alunos $(99,4 \%)$ responderam às perguntas e assinaram o TCLE. A amostra corresponde a $71,13 \%$ do total de alunos matriculados (478 acadêmicos) no curso de Medicina da Furb.

Foram aplicados dois questionários anônimos de autorresposta, um para coleta de dados sociodemográficos, psicossociais e uso de álcool e substâncias, e outro para rastreamento de transtornos mentais comuns (TMC). O primeiro, elaborado pelos autores, continha dados socioeconômicos (semestre de estudo, idade, sexo, etnia, estado civil, procedência, espiritualidade, religião, renda familiar e renda própria), informações sobre o curso e aprendizagem (motivo de escolha, satisfação com a escolha da profissão, pensamento de desistência, reprovação em matérias, carga horária média diária de aulas, carga horária média diária de estudos extraclasse, horas de lazer por dia, sentimento em relação às horas de lazer, número de faltas por semana e realização de plantão), fontes de tensão (cobrança pessoal, pressão social e de familiares e profissionais), redes de apoio (morar sozinho, com a família ou amigos, relação com os pais, dificuldade para fazer amigos, sentimento de rejeição por amigos ou outros da mesma idade e sentimento de que recebe apoio emocional necessário) e história médica pregressa (doença psiquiátrica diagnosticada por médico e medicação usada, história de tratamento medicamentoso psicoativo sem receita médica e realização de psicoterapia). A frequência de uso de álcool e outras substâncias foi avaliada por meio de questões referentes ao uso, nos últimos três meses, de álcool, tabaco, maconha, cocaína, crack, anfetamina, inalantes, sedativos, alucinógenos, opioides e outras substâncias. Para o rastreamento de TMC, foi aplicado o Self-Reporting Questionnaire-20 (SRQ-20), que é o instrumento elaborado pela Organização Mundial da Saúde para este fim ${ }^{10}$. Esse instrumento, na versão em português, é formado por 20 perguntas. Por meio dele avaliam-se transtornos não psicóticos (ansiedade, depressão e sintomas somatoformes), de modo que a presença dos sintomas avaliados indica os casos suspeitos de $\mathrm{TMC}^{10}$.

$\mathrm{Na}$ análise dos dados, os alunos foram classificados em dois grupos de acordo com a pontuação no $S R Q-20$ : (a) casos não suspeitos de TMC - homens com pontuação inferior ou igual a cinco e mulheres com pontuação inferior ou igual a sete; (b) casos suspeitos de TMC - homens com pontuação superior ou igual a seis e mulheres com pontuação superior ou igual a oito $^{5}$. Nesses pontos de corte, a sensibilidade e especificidade do $S R Q-20$ para homens são $89 \%$ e $81 \%$, enquanto para mulheres são $86 \%$ e $77 \%$, respectivamente ${ }^{11}$.
Os indivíduos considerados casos suspeitos de TMC foram comparados aos demais em relação aos dados obtidos pelo questionário sociodemográfico, psicossocial e uso de álcool e outras substâncias, evidenciando-se as características mais presentes nos casos suspeitos de TMC. Os dados foram inseridos numa planilha eletrônica do programa Microsoft Excel ${ }^{\circledR} 2013$ e analisados com uso do programa BioEstat, versão 5.3. Na análise dos dados foi usado o teste estatístico qui-quadrado de Pearson por análise bivariada e contagem de frequências. Foram consideradas variáveis significantes aquelas cuja associação identificada teve nível de significância (p) inferior a 0,05. O trabalho foi aprovado pelo Comitê de Ética na Pesquisa em Seres Humanos (CEPH) da Universidade Regional de Blumenau em 16 de dezembro de 2016 (Parecer ${ }^{\circ}$ 1.872.361)

\section{RESULTADOS}

A maioria da amostra era feminina (66,5\%), 57,9\% tinham entre 20 e 23 anos, e 54,1\% indicaram seguir alguma religião (Tabela 1). Na distribuição entre os ciclos, a maioria dos alunos pertencia ao ciclo básico e ao ciclo clínico, 71,1\% informaram deixar de assistir, em média, a menos de uma aula por semana, 53,2\% realizam, no mínimo, quatro horas semanais de atividade extraclasse. $46,3 \%$ informaram morar sozinhos, $18,6 \%$ com companheiro(a) e 35,1\% com a família. Dessa amostra, 92,6\% afirmaram não apresentar sintomas de depressão maior, $85 \%$ informaram não ter consumido maconha, $76,8 \%$ não consumiram tabaco e $92 \%$ consumiram álcool nos três meses que antecederam a aplicação dos questionários. Nenhum desses fatores, como mostra a Tabela 1, apresentou associação significante com o desenvolvimento de transtornos mentais comuns.

A prevalência de transtorno mental comum (TMC) na população estudada foi de 50,9\%, o que corresponde a 173 alunos classificados como grupo suspeito de TMC. A Tabela 2 apresenta associações entre prevalência de TMC e fatores acadêmicos e sociais. Cerca de $60,6 \%$ da amostra nunca pensaram em largar o curso. Sobre a carga horária semanal de atividades extracurriculares, $64,4 \%$ dos alunos tinham de 8 horas a 14 horas. Em relação às horas de lazer, 44,8\% apresentaram menos de duas horas de lazer por semana, e 66\% se sentem insatisfeitos com as horas de lazer; $87,2 \%$ opinaram que a carga horária de atividades acadêmicas é extensa. A competitividade foi indicada por $85,1 \%$ dos estudantes. Não sentir dificuldades em fazer amigos foi indicado por $73,7 \%$ da amostra. Cerca de $77,2 \%$ dos alunos não sentem rejeição pelos amigos ou outras pessoas da mesma faixa etária, e 66,8\% sentem que recebem apoio emocional necessário. 
TABELA 1

Distribuição dos estudantes segundo indicadores sociais, acadêmicos e de condições de saúde mental em relação às frequências de transtornos mentais comuns ( $p>0$,05). Blumenau (SC), 2017

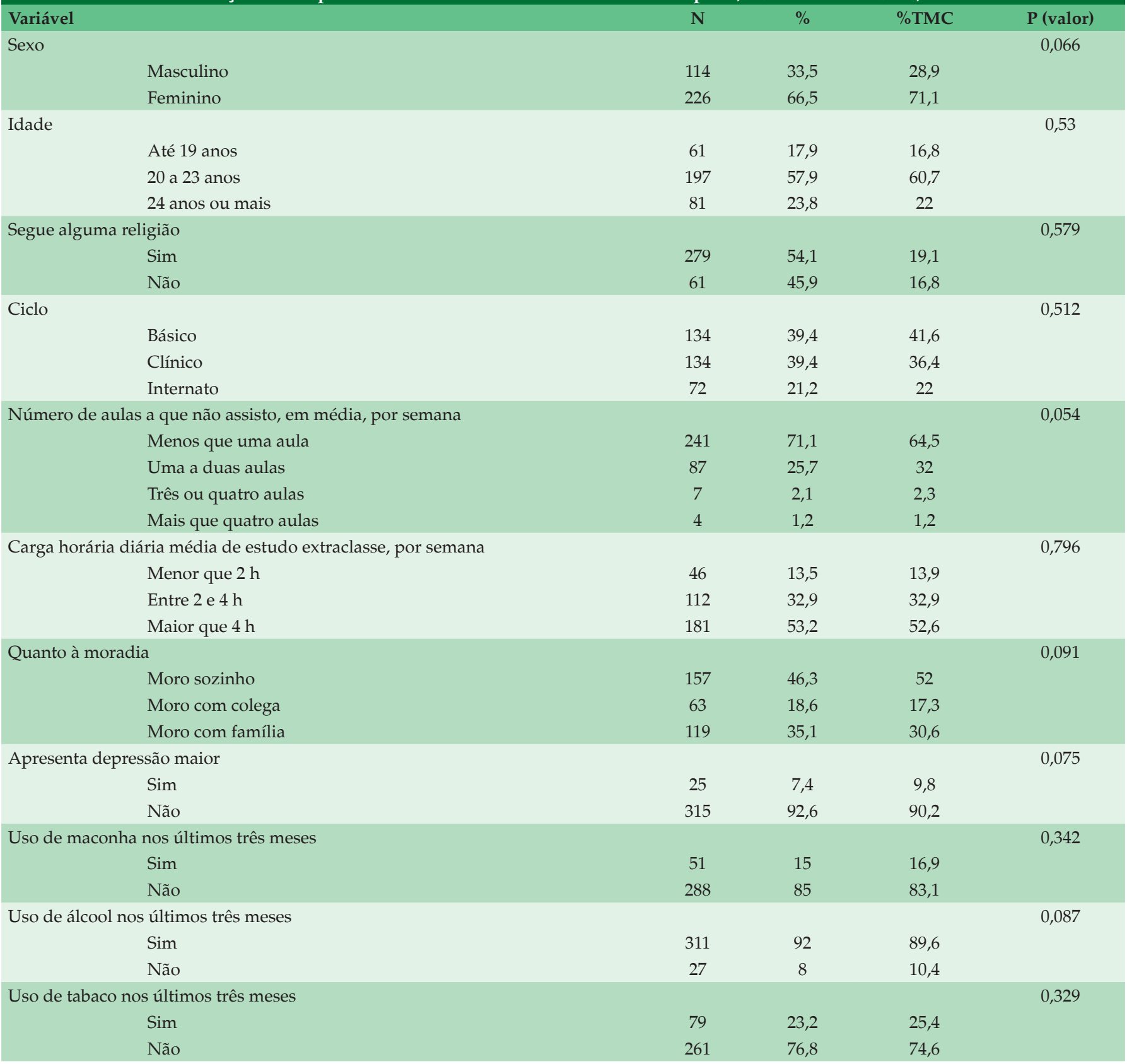

Fonte: Elaboração dos autores, 2017.

Considerando-se que todos os fatores apresentados na Tabela 2 apresentaram associação estatística significante $(p<0,05)$, a análise por qui-quadrado mostrou que foram encontrados proporcionalmente mais estudantes com transtornos mentais comuns entre aqueles que nunca pensaram ou não pensam mais em abandonar o curso, aqueles com cargas horárias diárias de atividades acadêmicas superiores a oito horas, tinham menos de duas horas de lazer por dia, mostraram insatisfação com as horas disponíveis para lazer, consideraram ter cargas horárias extensas de atividades, são competitivos, sentiam dificuldade para fazer amigos, sentiam rejeição por amigos de mesma faixa etária e não sentiam receber apoio emocional necessário. 


\begin{tabular}{|c|c|c|c|c|c|}
\hline $\begin{array}{l}\text { Distribuição dos estudantes seg } \\
\text { frequências de transtornos }\end{array}$ & $\begin{array}{l}\text { TABELA } 2 \\
0 \text { indicadores acac } \\
\text { tais comuns }(\mathrm{p}<\mathrm{C}\end{array}$ & $\begin{array}{l}\text { os e s } \\
\text { Blum }\end{array}$ & em re & & \\
\hline Variável & & $\mathbf{N}$ & $\%$ & \%TMC & P (valor) \\
\hline Já pensou em abandonar o curso & & & & & $<0,01$ \\
\hline & Não, nunca & 206 & 60,6 & 45,1 & \\
\hline & Sim, mas não mais & 107 & 31,5 & 43,4 & \\
\hline & Sim, ainda penso & 27 & 7,9 & 11,6 & \\
\hline $\begin{array}{l}\text { Carga horária diária média de aulas/atividades } \\
\text { extracurriculares por dia }\end{array}$ & & & & & 0,035 \\
\hline & Entre $4 \mathrm{~h}$ e $8 \mathrm{~h}$ & 72 & 21,2 & 23,1 & \\
\hline & Entre $8 \mathrm{~h}$ e $12 \mathrm{~h}$ & 219 & 64,4 & 58,4 & \\
\hline & Mais que $12 \mathrm{~h}$ & 49 & 14,4 & 18,5 & \\
\hline Horas de lazer por dia & & & & & 0,004 \\
\hline & Menos de $1 \mathrm{~h}$ & 77 & 22,7 & 28,3 & \\
\hline & Menos de $2 \mathrm{~h}$ & 152 & 44,8 & 46,8 & \\
\hline & Menos de $3 \mathrm{~h}$ & 81 & 23,9 & 20,2 & \\
\hline & Mais que $3 \mathrm{~h}$ & 29 & 8,6 & 4,6 & \\
\hline Como você se sente quanto às horas disponíveis para lazer & & & & & $<0,01$ \\
\hline & Satisfeito & 80 & 23,7 & 14,5 & \\
\hline & Indiferente & 34 & 10,1 & 9,9 & \\
\hline & Insatisfeito & 223 & 66 & 75,6 & \\
\hline Carga horária extensa & & & & & 0,047 \\
\hline & Sim & 294 & 87,2 & 90,8 & \\
\hline & Não & 43 & 12,8 & 9,2 & \\
\hline Competitividade & & & & & 0,003 \\
\hline & Sim & 286 & 85,1 & 90,8 & \\
\hline & Não & 50 & 14,9 & 9,2 & \\
\hline Sente dificuldade em fazer amigos & & & & & $<0,01$ \\
\hline & Sim & 89 & 26,3 & 37 & \\
\hline & Não & 250 & 73,7 & 63 & \\
\hline Sente rejeição por amigos ou outros da mesma faixa etária & & & & & $<0,01$ \\
\hline & Sim & 77 & 22,8 & 32,6 & \\
\hline & Não & 260 & 77,2 & 67,4 & \\
\hline Sente que recebe o apoio emocional necessário & & & & & $<0,01$ \\
\hline & Sim & 223 & 66,8 & 55,6 & \\
\hline & Não & 111 & 33,2 & 44,4 & \\
\hline
\end{tabular}

Fonte: Elaboração dos autores, 2017.

A Tabela 3 apresenta associações entre prevalência de TMC e indicadores de saúde mental. A maioria dos alunos afirmou existência de cobrança pessoal $(94,4 \%)$, pressão social $(73,3 \%)$, dos professores e profissionais da área (72,4\%), mas inexistência de pressão familiar (57,6\%); 77,6\% dos participantes afirmaram não apresentar transtornos psiquiátricos, e 91,5\% não apresentaram transtorno de ansiedade generalizada; 80,8\% dos participantes informaram não utilizar medicação psicoativa; $87,6 \%$ não fazem uso de antidepressivo; $67,8 \%$ dos participantes afirmaram nunca ter frequentado psicoterapia; e 50,6\% informaram ter feito uso de drogas recreativas nos três meses anteriores à data da pesquisa. Os fatores apresentados na Tabela 3 apresentaram associação estatística significante $(\mathrm{p}<0,05)$, de modo que a análise por qui-quadrado mostrou que foram encontrados proporcionalmente mais participantes com transtornos mentais comuns entre aqueles que afirmaram haver cobrança pessoal, pressão social, pressão dos professores e profissionais da área, pressão familiar, doença psiquiátrica diagnosticada por médico, transtorno de ansiedade generalizada, fazer uso de medicação psicoativa, usar antidepressivo, frequentar atualmente ou ter abandonado psicoterapia e ter feito uso de drogas recreativas nos três meses anteriores à data da pesquisa. 
TABELA 3

Distribuição dos estudantes segundo indicadores de condições de saúde mental em relação às frequências de transtornos mentais comuns (p < 0,05). Blumenau (SC), 2017

\begin{tabular}{|c|c|c|c|c|c|}
\hline Variável & & $\mathbf{N}$ & $\%$ & $\%$ TMC & P (valor) \\
\hline \multirow[t]{3}{*}{ Cobrança pessoal } & & & & & 0,0002 \\
\hline & Sim & 320 & 94,4 & 98,8 & \\
\hline & Não & 19 & 5,6 & 1,2 & \\
\hline \multirow[t]{3}{*}{ Pressão social } & & & & & 0,001 \\
\hline & Sim & 247 & 73,3 & 80,9 & \\
\hline & Não & 90 & 26,7 & 19,1 & \\
\hline & Sim & 244 & 72,4 & 80,9 & \\
\hline & Não & 93 & 27,6 & 19,1 & \\
\hline \multirow[t]{3}{*}{ Pressão familiar } & & & & & 0,001 \\
\hline & Sim & 143 & 42,4 & 50,9 & \\
\hline & Não & 194 & 57,6 & 49,1 & \\
\hline $\begin{array}{l}\text { Apresenta doença psiquiátrica diagnosticada por } \\
\text { médico }\end{array}$ & Não & 263 & 77,6 & 70,5 & \\
\hline \multirow[t]{3}{*}{ Apresenta transtorno de ansiedade generalizada } & & & & & 0,005 \\
\hline & Sim & 29 & 8,5 & 12,7 & \\
\hline & Não & 311 & 91,5 & 87,3 & \\
\hline \multirow[t]{3}{*}{ Faz uso de medicação psicoativa } & & & & & 0,001 \\
\hline & Sim & 65 & 19,2 & 26,2 & \\
\hline & Não & 273 & 80,8 & 73,8 & \\
\hline \multirow[t]{2}{*}{ Uso de antidepressivo } & & & & & 0,004 \\
\hline & Sim & 42 & 12,4 & 17,3 & \\
\hline \multirow[t]{3}{*}{ Uso de drogas recreativas nos últimos três meses } & & & & & 0,02 \\
\hline & Sim & 171 & 50,6 & 67,8 & \\
\hline & Não & 167 & 49,4 & 79 & \\
\hline
\end{tabular}

Fonte: Elaboração dos autores, 2017.

\section{DISCUSSÃO}

A prevalência de transtornos mentais comuns entre os estudantes de Medicina em universidades do Brasil mostrou-se variável nos estudos analisados ${ }^{1,5-7,12,13}$. Na Universidade Regional de Blumenau (Furb), a prevalência de casos suspeitos de TMC foi de 50,9\%, o que representa maior taxa em relação aos dados encontrados nos estudos analisados: pesquisas realizadas na Universidade Federal da Paraíba (UFPB), no cur- so de Medicina de Botucatu (SP) e na Universidade Federal do Espírito Santo (Ufes) encontraram prevalências de 33,6\%, $44,7 \%$ e $37,1 \%$, respectivamente ${ }^{1,5,6}$.

Características da realidade da vida universitária e do processo de ensino têm sido relatadas como fontes geradoras de conflitos, afetando negativamente a saúde mental e o desempenho acadêmico dos alunos ${ }^{7}$. Quando comparamos a prevalência dos estudantes universitários com a da população 
geral, o valor encontrado é maior em relação às taxas populacionais, usando-se o mesmo instrumento de rastreamento $(S R Q-20)^{1,12,13,14}$. Um estudo populacional realizado na cidade de Olinda (PE) evidenciou que 35\% da amostra tinham TMC; já em São Paulo (SP) e Santa Cruz do Sul (RS), pesquisas feitas em programas de saúde da família encontraram que 24,95\% e $38 \%$ da amostra, respectivamente, eram classificados como casos suspeitos ${ }^{8,12,13}$. O resultado encontrado possivelmente se deve ao fato de os estudantes de Medicina pertencerem a um grupo vulnerável a situações estressantes ${ }^{14}$.

$\mathrm{O}$ ambiente adverso da faculdade de Medicina faz com que o estudante experimente três fases psicológicas na graduação ${ }^{14}$ : euforia, quando há percepção de onipotência; decepção, que se inicia nos dois primeiros anos do curso, quando há alteração dos hábitos cotidianos; a última fase, que ocorre no internato, reflete um período de adaptação e grande competitividade pela residência ${ }^{14}$. Estudos em outras universidades de Medicina do País mostraram associação entre os ciclos básico e clínico do curso e maiores prevalências de TMC, em relação a um menor valor no período do internato ${ }^{1,5}$. A menor prevalência de transtornos mentais comuns nos dois últimos anos da faculdade pode ser explicada por conta de fatores protetores, como, por exemplo, idade maior que 20 anos, maior maturidade e habilidade para lidar com os estressores ${ }^{5}$.

Uma característica do curso de Medicina é a carga horária extensa de aulas ${ }^{15}$. Além disso, os acadêmicos de Medicina ainda dedicam parte de seu tempo livre a outras atividades, como estudo individual, ligas acadêmicas, iniciação científica, monitorias e estágios ${ }^{15}$. Foi demonstrado que o menor número médio de horas de lazer por dia é associado a maior frequência de TMC, indicando que este pode ser um fator de risco. A prevalência de TMC entre o grupo que afirma ter menos de uma hora de lazer em média por dia foi 2,3 vezes maior que a encontrada no grupo que respondeu ter três horas de lazer em média por dia.

A história médica pregressa de doença psiquiátrica mostrou relação com TMC, sendo transtornos de ansiedade generalizada e depressão maior os mais presentes, com prevalências de $8,5 \%$ e $7,4 \%$, respectivamente. Pode-se supor que parte dos acadêmicos que está no grupo suspeito de TMC buscou assistência médica, porque tem diagnósticos específicos de transtornos mentais. Dentro da população de casos suspeitos de TMC, 122 estudantes (70,5\%) responderam não ter doença psiquiátrica diagnosticada. Neste grupo, para aqueles que não realizaram avaliação psiquiátrica recente, caberia investigação por profissionais da saúde mental ${ }^{6}$.

Transtorno mental comum demonstrou estar relacionado com maior uso de antidepressivos e ansiolíticos, sendo que apenas 28,6\% do grupo que usa antidepressivos não apresentaram TMC. Desta forma, apesar do uso da medicação, 71,4\% dos estudantes ainda estavam em sofrimento mental. Neste sentido, má adesão ao tratamento e desafios sociais crônicos relacionados ao curso de Medicina poderiam explicar a ausência de resposta ao uso da medicação ${ }^{16}$. Ter concluído tratamento com psicoterapeuta mostrou ser fator protetor de TMC, de acordo com estudos que mostraram eficácia da psicoterapia no tratamento da depressão de leve a moderada ${ }^{17}$.

Indivíduos insatisfeitos com o suporte emocional fornecido pelo grupo social, família e amigos são mais suscetíveis a transtornos mentais, principalmente sintomas depressivos ${ }^{18,19}$. Sentir que não recebe o apoio emocional que necessita mostrou ser fator de risco para TMC, como apresentado na Tabela 2, similarmente ao encontrado em estudos anteriores com alunos de Medicina ${ }^{1,5,6}$. Muitos acadêmicos deixam o lar familiar quando iniciam a graduação em Medicina ${ }^{20}$. Dificuldade para fazer amigos e se sentir rejeitado levam a uma deficiência na composição da nova rede de apoio local ${ }^{1,6}$ e demonstraram relação com maiores prevalências de TMC na Furb. O grupo de alunos que respondeu "sentir dificuldade para fazer amigos" apresentou maior proporção de participantes com TMC do que o grupo que respondeu "não", com 71,9\% e 43,6\%, respectivamente. Morar com a família ou amigos deveria fornecer uma rede de apoio protetora para desenvolvimento de transtorno mental ${ }^{21}$, mas, no presente, estudo morar sozinho não mostrou associação significativa com prevalência de TMC.

Mostraram-se com maior prevalência de TMC os alunos que classificaram como fontes de tensão carga horária extensa, competitividade, cobrança pessoal, pressão social, familiar, de professores e profissionais da área. A intensa demanda e competição durante o processo de educação médica colabora para produzir estresse nos estudantes. Há uma alteração de autoimagem, pois o aluno, antes reconhecido como capaz e bem-sucedido, se depara com falhas e resiste em aceitá-las. Além disso, há uma crescente responsabilidade em virtude do papel que o médico desempenha ${ }^{20}$.

O uso de álcool e outras substâncias (tabaco, maconha, cocaína, crack, anfetaminas, êxtase, inalantes, hipnóticos/sedativos, alucinógenos e opioides) mostrou associação significante com a presença de TMC, o que indica constituir um fator de risco. Já o consumo isolado de cada substância não demonstrou relação estatística significante, talvez relacionada à baixa frequência de uso de cada substância individual. Na população estudada, 92\% responderam ter usado álcool nos últimos três meses, valor superior ao de outros cursos de Medicina no Brasil $^{22,23}$. No curso de Medicina da Universidade Federal de Uberlândia (MG), 66,34\% afirmaram consumir álcool22. Já en- 
tre estudantes de Medicina de Salvador (BA), 87,2\% responderam ter consumido alguma bebida alcoólica nos últimos 12 meses $^{23}$. A prevalência do uso de maconha na amostra foi de 15\%, compatível com estudos realizados em oito escolas médicas do Estado de São Paulo, no qual os valores encontrados variaram de $6 \%$ a $16 \%{ }^{24}$.

O presente estudo não encontrou significância entre gênero e prevalência de TMC, resultado que reproduz o encontrado na literatura pesquisada, na qual os sexos feminino e masculino apresentam prevalências sem diferença estatística relevante ${ }^{4-7}$. Neste estudo, nenhuma religião demonstrou relação significativa com TMC (Tabela 1), diferentemente de outros estudos, que identificaram associação entre não seguir alguma religião com maior prevalência de $\mathrm{TMC}^{5}$ ou com a religião espírita, considerada fator de risco quando comparada a outras ou a nenhuma religião ${ }^{1}$.

Um possível viés do estudo foi o número reduzido de estudantes do internato que responderam ao questionário, devido à dificuldade de encontrá-los, por terem poucas aulas teóricas em salas de aula ou estarem em estágio fora da cidade. Outro ponto a enfatizar é que, embora o instrumento $S R Q-20$ tenha alta sensibilidade e especificidade para rastreamento de casos de TMC, o diagnóstico psiquiátrico formal deve ser realizado por profissional da saúde mental ${ }^{11}$. Como características do estudo transversal, as variáveis e o desfecho são concomitantemente estudados, o que impossibilita inferir sobre a causalidade das associações analisadas. Todavia, esses estudos norteiam sentidos em que a variável e o desfecho estudado se associam ${ }^{6}$.

\section{CONCLUSÃO}

Os resultados encontrados indicam maior prevalência de casos suspeitos de TMC na população estudada do que em outros cursos de Medicina brasileiros e do que na população geral. Os acadêmicos que têm história médica pregressa de doença psiquiátrica, que não consideram ter uma rede de apoio psicológica adequada e que não têm bons relacionamentos com pessoas da mesma idade têm maior risco de ter TMC. Pode-se concluir também que os estudantes em sofrimento mental usam mais medicações psicoativas e mais álcool e outras substâncias. Essas informações fornecem subsídios para ações de prevenção e cuidado da saúde mental. Propõe-se investigar características do currículo do curso de Medicina da Furb, em especial do internato, que possivelmente estejam contribuindo para manutenção de altas prevalência de TMC, mesmo na presença de fatores protetores. Recomenda-se a estruturação de um programa de apoio à saúde mental dos estudantes, a fim de melhorar a qualidade de vida e a formação médica.

\section{ORGANIZAÇÃO DE FOMENTO À PESQUISA}

Fundação Universidade Regional de Blumenau (Furb)

\section{REFERÊNCIAS}

1. Fiorotti K, Rossoni R, Borges L, Miranda A. Transtornos mentais comuns entre os estudantes do curso de medicina: prevalência e fatores associados. J Bras. Psiquiatr. [online]. 2010. 59(1). [ capturado em 1 out. 2017]; 17-23. Disponível em: http: / / www.scielo.br/scielo.php?pid=S0047-20852010000100003\&script=sci_abstract\&tlng=pt.

2. Lucchese R, Sousa K, Bonfin S, Vera I, Santana F. Prevalência de transtorno mental comum na atenção primária. Acta Paul Enferm. [online]. 2017. 27(3). [ capturado 1 out. 2017]; 200-207. Disponível em: http://www.scielo.br/ scielo.php?pid=S0103-21002014000300200\&script $=$ sci_ abstract\&tlng=pt.

3. Brasil. Ministério da Saúde. Diretrizes do NASF: Núcleo de Apoio a Saúde da Família. Série A. Normas e Manuais Técnicos, Cadernos de Atenção Básica, n. 27. Brasília, 2010 p. 152.

4. Ferreira C,Kluthcovsky A, Cordeiro T. Prevalência de Transtornos Mentais Comuns e Fatores Associados em Estudantes de Medicina: um Estudo Comparativo. Revista Brasileira de Educação Médica. [online]. 2016. 40(2). [ capturado 1 out. 2017]; 268-277. Disponível em: http:/ /www.scielo. br / scielo.php?pid=S0100-55022016000200268\&script=sci_ abstract\&tlng=pt

5. Rocha E, Sassi A. Transtornos mentais menores entre estudantes de medicina. Revista Brasileira de Educação Médica [online]. 2013. 37(2) [ capturado 1 out. 2017]; 210-216. Disponível em: http:/ / www.scielo.br/scielo.php?pid=S0100$-55022013000200008 \&$ script=sci_abstract\&tlng=pt

6. Lima M, Domingues M, Cerqueira A. Prevalência e fatores de risco para transtornos mentais comuns entre estudantes de medicina. Revista de Saúde Pública [online]. 2006. 40(6) [ capturado 1 Out. 2017]; 1035-1041. Disponível em: https:/ / repositorio.unesp.br/bitstream/handle/11449/12489/ S0034-89102006000700011.pdf?sequence $=1$

7. Facundes V, Ludermir A. Common mental disorders among healthcare students. Revista Brasileira de Psiquiatria [online]. 2005. 27(3). [ capturado 1 out. 2017]; 194-200. Disponível em: http://www.scielo.br/scielo.php?pid=S1516$-44462005000300007 \&$ script=sci_abstract\&tlng=pt

8. Ludermir A, Melo Filho D. Condições de vida e estrutura ocupacional associadas a transtornos mentais comuns. Rev. Saúde Pública [online]. 2002. 36(2). [ capturado 1 out. 2017]; 213-221 Disponível em: http://www.scielo. br /scielo.php?pid=S0034-89102002000200014\&script=sci abstract\&tlng $=\mathrm{pt}$ 
9. Zonta R, Robles A, Grosseman S. Estratégias de enfrentamento do estresse desenvolvidas por estudantes de Medicina da Universidade Federal de Santa Catarina. Revista Brasileira de Educação Médica [Internet]. 2006. 30(3) [ capturado 1 Out. 2017]; 147-153. Disponível em: http://www.scielo.br/scielo.php?pid=S0100-55022006000300005\&script=sci_abstract\&tlng=pt

10. Santos KOB, Araújo T, Pinho PS, Silva AC. Avaliação de um instrumento de mensuração de morbidade psíquica: estudo de validação do Self-ReportingQuestionnaire (SRQ-20). Rev Baiana Saúde Pública Miolo [online]. 2010. 34(3). [capturado 1 out. 2017]; 544-560. Disponível em: http:/ / files. bvs.br/upload/S/0100-0233/2010/v34n3/a1881.pdf

11. Mari J, Williams P. A validity study of a psychiatric screening questionnaire (SRQ-20) in primary care in the city of Sao Paulo. British Journal of Psychiatry [online]. 1986. [ capturado 1 out. 2017]; 23-26. Disponível em: https:/ / www.ncbi.nlm.nih.gov/pubmed/3955316

12. Maragno L, Goldbaum M, Gianini R, Novaes H, César C. Prevalência de transtornos mentais comuns em populações atendidas pelo Programa Saúde da Família (QUALIS) no Município de São Paulo, Brasil. Cad. Saúde Pública [Internet]. 2006. 22(8). [capturado 1 out. 2017]; 1639-1648. Disponível em: http://www.scielo.br/scielo.php?script=sci_ arttext\&pid=S0102-311X2006000800012.

13. Gonçalves D, Kapczinski F. Transtornos mentais em comunidade atendida pelo Programa Saúde da Família. Cad. Saúde Pública [online]. 2008. 24(7). [cited 1 October 2017];1641-1650. Disponível em: https://www.scielosp. org/pdf/csp/v24n7/19.pdf

14. Abrão C, Coelho E, Passos L, de Rezende C. Prevalência de sintomas depressivos entre estudantes de medicina da Universidade Federal de Uberlândia. Revista Brasileira de Educação Médica [online]. 2008. 32(3). [capturado 1 out. 2017]; 315-323. Disponível em: http://www.scielo.br/ $\mathrm{pdf} / \mathrm{rbem} / \mathrm{v} 32 \mathrm{n} 3 / \mathrm{v} 32 \mathrm{n} 3 \mathrm{a} 06$

15. Avaliação da qualidade de vida do estudante de medicina e da influência exercida pela formação acadêmica: São Paulo; 2008. Doutorado [tese] - Universidade de São Paulo.

16. Fleck MPA, Lafer B, Sougey EB, Del Porto JA, Brasil MA, Juruena MF. Diretrizes da Associação Médica Brasileira para o tratamento da depressão (versão integral) Ver BrasPsiquiatr [online]. 2003. 25(2). [ capturado 1 out. 2017]; 114-122. Disponível: http://www.scielo.br/ scielo.php?pid=S1516-44462003000200013\&script=sci_ abstract\&tlng $=\mathrm{pt}$
17. Persons J, Thase ME, Crits-Christoph P. The Role of Psychotherapy in the Treatment of Depression. Archives of General Psychiatry [online]. 1996. 53(4). [capturado 1 out. 2017]; 283-290. Disponível: https://jamanetwork.com/ journals/jamapsychiatry/article-abstract/497557

18. Krause N, Liang J, Yatomi N. Satisfaction with social support and depressive symptoms: A panel analysis. Psychology and aging [online]. 1989. 4(1). [ capturado 1 out. 2017]; 88-97. Disponível em: http:/ / psycnet-apa-org.ez71.periodicos.capes.gov.br/fulltext/1989-26115-001.html

19. Rubin C, Rubenstein J, Stechler G, Heeren T, Halton A, Housman D, et al. Depressive affect in "normal" adolescents: Relationship to life stress, family, and friends. American Journal of Orthopsychiatry. [online] 1992. 62 (3) [capturado 1 out. 2017]; 430-441. Disponível em: http:/ / psycnet.apa.org/buy/1992-43215-001

20. Notman M, Salt P, Nadelson C. Stress and adaptation in medical students: Who is most vulnerable? Comprehensive Psychiatry. [online] 1984. 25(3) [capturado 1 out 2017]; 355-366. Disponível em: https://www-sciencedirect.ez71.periodicos.capes.gov.br/science/article/ pii/0010440X84900683?via\%3Dihub

21. Gligio J. Bem-estar emocional em estudantes universitários. Campinas; 1976. Doutorado [tese] - Universidade Estadual de Campinas.

22. Paduani G, Barbosa G, Morais J, Pereira J, Almeida M, Prado M, et al. Consumo de álcool e fumo entre os estudantes da Faculdade de Medicina da Universidade Federal de Uberlândia. Revista Brasileira de Educação Médica. 2008. 32(1). [capturado 1 out. 2017]; 66-74. Disponível em: http:/ / www.scielo.br/pdf/rbem/v32n1/09.pdf

23. Lemos KM, Neves NMBC, Kuwano AY, Tedesqui G, Bitencourt AGV, Neves FBCS, et al. Uso de substâncias psicoativas entre estudantes de Medicina de Salvador (BA). Rev. Psiq. Clín. [online] 2007. 34(3). [capturado 1 out. 2017]; 118-124. Disponível em: http:/ / www.scielo.br/pdf/rpc/ v34n3/a03v34n3.pdf

24. Kerr-Corrêa F, Andrade A, Bassit A, Boccuto N. Uso de álcool e drogas por estudantes de medicina da Unesp. RevBrasPsquiatr. 1999. 21(2). [capturado 1 out. 2017]; 95 - 100. Disponível em: http://www.scielo.br/pdf/rbp/v21n2/ v21n2a05.pdf 


\section{CONTRIBUIÇÃO DOS AUTORES}

Eduardo Otávio Grether: delineamento da pesquisa, coleta, análise e escrita do manuscrito. Mateus Ciola Becker: delineamento da pesquisa, coleta, análise e escrita do manuscrito. Helena Medina Menezes: redação, adequação e submissão do manuscrito. Carlos Roberto de Oliveira Nunes: orientador do estudo.

\section{CONFLITO DE INTERESSES}

Este estudo não apresenta conflito de interesses.

ENDEREÇO PARA CORRESPONDÊNCIA

R. São Paulo, 2171 - Itoupava Seca, Blumenau - SC, 89030-001 